\title{
Evolution of rhizobial symbiosis islands through insertion sequence-mediated deletion and duplication
}

\author{
Haruka Arashida (D ${ }^{1}$, Haruka Odake ${ }^{1}$, Masayuki Sugawara ${ }^{1}{ }^{1}$, Ryota Noda ${ }^{1}$, Kaori Kakizaki ${ }^{1}$, Satoshi Ohkubo $\mathbb{D}^{1}$, Hisayuki Mitsui ${ }^{1}$, \\ Shusei Sato $\mathbb{D}^{1}$ and Kiwamu Minamisawa (iD ${ }^{1 \times}$ \\ (c) The Author(s) 2021
}

\begin{abstract}
Symbiosis between organisms influences their evolution via adaptive changes in genome architectures. Immunity of soybean carrying the Rj2 allele is triggered by NopP (type III secretion system [T3SS]-dependent effector), encoded by symbiosis island A (SymA) in B. diazoefficiens USDA122. This immunity was overcome by many mutants with large SymA deletions that encompassed T3SS (rhc) and $\mathrm{N}_{2}$ fixation (nif) genes and were bounded by insertion sequence (IS) copies in direct orientation, indicating homologous recombination between ISs. Similar deletion events were observed in $B$. diazoefficiens USDA110 and $B$. japonicum J5. When we cultured a USDA122 strain with a marker gene sacB inserted into the rhc gene cluster, most sucroseresistant mutants had deletions in nif/rhc gene clusters, similar to the mutants above. Some deletion mutants were unique to the $\operatorname{sac} B$ system and showed lower competitive nodulation capability, indicating that IS-mediated deletions occurred during free-living growth and the host plants selected the mutants. Among 63 natural bradyrhizobial isolates, 2 possessed long duplications (261-357 kb) harboring nif/rhc gene clusters between IS copies in direct orientation via homologous recombination. Therefore, the structures of symbiosis islands are in a state of flux via IS-mediated duplications and deletions during rhizobial saprophytic growth, and host plants select mutualistic variants from the resultant pools of rhizobial populations. Our results demonstrate that homologous recombination between direct IS copies provides a natural mechanism generating deletions and duplications on symbiosis islands.
\end{abstract}

The ISME Journal (2022) 16:112-121; https://doi.org/10.1038/s41396-021-01035-4

\section{INTRODUCTION}

Symbiotic organisms can co-evolve through adaptive changes in the organization of the functional elements in their genomes. For instance, in animal-bacteria symbiosis, the evolution of obligate symbiont bacteria with intercellular lifestyles has been accompanied by a marked reduction in genome size [1-3]. The best-studied plant-bacteria symbiosis involves legume plants and nitrogen-fixing bacteria called rhizobia [3, 4]. Rhizobia have repeated symbiotic phases (in the plant) and free-living phases (in the soil) [4, 5]. As facultative symbionts, rhizobia generally possess the distinct packages of symbiosis genes (symbiosis islands or symbiotic plasmids) within their genome $[3,4,6]$.

Major rhizobia in Alphaproteobacteria include species within the genera Bradyrhizobium, Azorhizobium, Sinorhizobium, Rhizobium, and Mesorhizobium [7]. Among them, Bradyrhizobium species are thought to be ancestral rhizobia, because Bradyrhizobium, which shows enormous species diversity (>800 species), nodulates primitive leguminous plants $[6,8,9]$.

Rhizobial symbiosis islands on the genomes of Bradyrhizobium [10-12], Azorhizobium [13], and Mesorhizobium [14-18] contain strain-specific symbiotic genes. Comparisons of Mesorhizobium genomes reveal different structures of symbiosis islands depending on geography and host plants [16-18]. In Bradyrhizobium, symbiosis islands include nod, nif, and rhc, which function in nodulation of roots, $\mathrm{N}_{2}$ fixation, and type III secretion system (T3SS), respectively.
The symbiosis islands contain conserved regions relevant to the above symbiotic genes and extremely mosaic regions containing insertion sequences (ISs) [10-12, 16-18]. However, little is known about how the symbiosis island structure changes depending on the strain.

ISs are simple mobile genetic elements that impact bacterial evolution including deleterious, neutral, or beneficial effects in bacteria [19]. Multiple copies of an identical IS element dispersed over a genome can promote various genomic rearrangements such as inversion, deletion, duplication, and fusion of two replicons [20,21]. However, the evolutionary roles of IS elements have been underestimated due to difficulty in their identification on bacterial genomes; (i) repetition of the same ISs on the genomes, and (ii) peculiar signatures of IS elements [22]. ISs are composed of one or two transposaseencoding genes and two terminal inverted repeats, generating two direct repeated sequences as target duplication at the border during transposition [23].

Cultivar-specific restriction of nodulation in soybean has a long history from the 1960s: several dominant genes (Rj2, Rj3, $R j 4$, and $R f g 1)$ in soybeans restrict nodulation with specific rhizobial strains [24-26]. Rj2-genotype soybeans restrict nodulation with Bradyrhizobium diazoefficiens USDA122 [24, 25]. Rhizobia secrete through type III secretion system nodule outer proteins (Nops), which impact positive, neutral, and negative

${ }^{1}$ Graduate School of Life Sciences, Tohoku University, 2-1-1 Katahira, Aoba-Ku, Sendai 980-8577, Japan. ${ }^{凶}$ email: kiwamu.minamisawa.e6@tohoku.ac.jp 


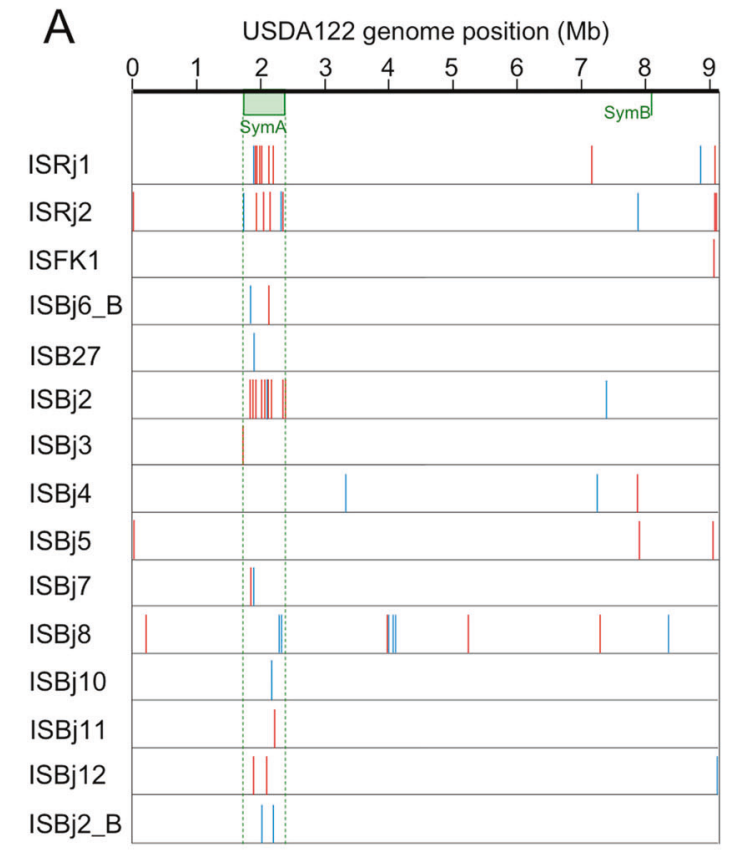

B Symbiosis island of USDA122 genome (Mb)
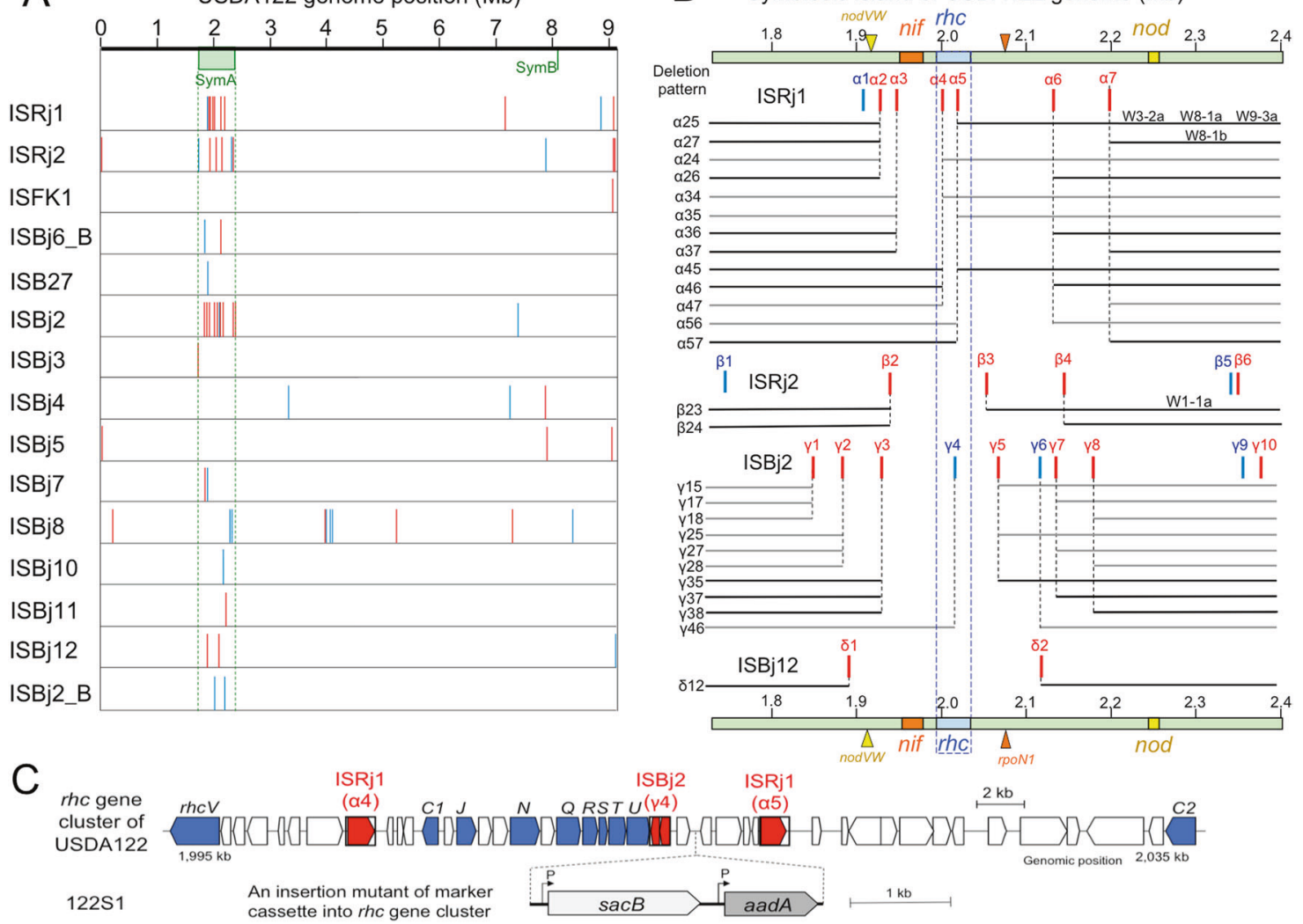

Fig. 1 The position of the insertion sequences (ISs) in the genome (A), symbiosis island A (B), and the rhc gene cluster (C) of Bradyrhizobium diazoefficiens USDA122. Red and blue vertical lines show ISs with their clockwise and anticlockwise directions on the genome, respectively. A IS position of 15 different ISs was identified on USDA122 genome. Symbiosis islands of A and B (SymA and SymB) were estimated based on USDA110 genome [10,11]. Each IS element was numbered along with the location of the SymA, such as $\alpha 2$. B Predicted deletion patterns between the ISs were estimated on the assumption that parts of the rhc genes encoding the T3SS machinery were deleted between the same IS elements with identical directions on the SymA of USDA122. The deletion patterns were named based on the IS position, such as $\alpha 25$. We included previous deletion patterns of $\alpha 25, \alpha 27$, and $\beta 23$ from the USDA122 mutants W3-2a, W8-1a, W9-3a, W8-1b, and W1-1a [31]. Bold black lines indicate the deletion patterns that were experimentally obtained. Orange, blue, and yellow squares show nif, rhc, and nod gene clusters on SymA, respectively. Yellow and red triangles indicate the positions of nodVW and rpoN1 genes, respectively (see text). C Genetic organization of the rhc gene cluster on the genomes of $B$. diazoefficiens USDA122 and its marker cassetteinsertion mutant $122 \mathrm{~S} 1$. The marker cassette containing $s a c B$ (sucrose sensitive gene) and aadA (streptomycin and spectinomycin resistant gene) with its own constitutive promoter $(P)$, was inserted in an intergenic region in the rhc gene cluster.

effects on symbiosis depending on combinations between rhizobia and host plants [27-29]. Although most Nops diminish plant defense responses during rhizobial infection [27-29], Sinorhizobium fredii NopP oppositely elicited soybean defense responses [30]. Sugawara and co-workers found that bradyrhizbial NopP is a causal T3SS effector to induce symbiotic incompatibility with Rj2-genotype soybeans via effectortriggered immunity [31-33]. Zhao et al. [34] reported adaptive evolution of symbiotic compatibility by IS insertion into type III and nopP genes by incompatible combinations between wildtype Sinorhizobium fredii and soybeans.

Sugawara et al. [31] also found five mutants that overcame NopP-Rj2 incompatibility due to partial genome deletions in Bradyrhizobium diazoefficiens USDA122. Some of these mutants had deleted T3SS gene ( $r h c)$ clusters on the symbiosis island; in these mutants the resultant lack of the T3SS machinery prevented NopP secretion [31]. Other mutants, in which both nif and rhc genes were deleted, showed no $\mathrm{N}_{2}$-fixing activity [31] and were parasitic mutants [35].

Here, we studied the dynamics of symbiosis island structures based on IS elements and Rj2 incompatibility to reveal the mechanisms behind the generation of symbiosis island variations in Bradyrhizobium, and their involvement in phenotypic drift for mutualistic and parasitic behaviors.

\section{RESULTS}

Bioinformatic prediction of IS-mediated deletions

In a previous study, we determined that two ISs in direct orientation on symbiosis island $A$ (SymA) of $B$. diazoefficiens USDA122 were involved in three types of deletions in the USDA122 genome [31]. Here, we examined the IS element distributions in USDA122 SymA to predict and confirm the modes of IS-mediated deletion.

The positions of symbiosis islands on the USDA122 genome [36] were determined using the low $\mathrm{G}+\mathrm{C}$ content of symbiosis islands and their position relative to the Val-tRNA gene in the genome of strain USDA110 [10, 11]. The major symbiosis island SymA (671 $\mathrm{kb})$, which includes nif, rhc, and nod genes, was located in the USDA122 genome at coordinates 1,730,003-2,401,617 bp (Fig. 1A).

We then searched the USDA122 genome for 21 IS elements (ISRj1, ISRj2, ISFK1, IS1632, ISBj6_B, ISB27, ISBj2 to ISBj12, ISBj7_B, ISBj5_B, ISBj2_B, and IS1631) that were previously identified in the genomes of strains USDA110 [11] and NK6 [37]. When we subjected the USDA122 genome to a BlastN search with the IS elements as query sequences, $A$ total of 63 copies of 15 different IS elements were found, 37 copies of which were located within SymA (Fig. 1A; Table S1).

Seven copies of ISRj1, named a1-a7, and six copies of ISRj2, named $\beta 1-\beta 6$, resided on SymA on USDA122 genome (Fig. 1B). 

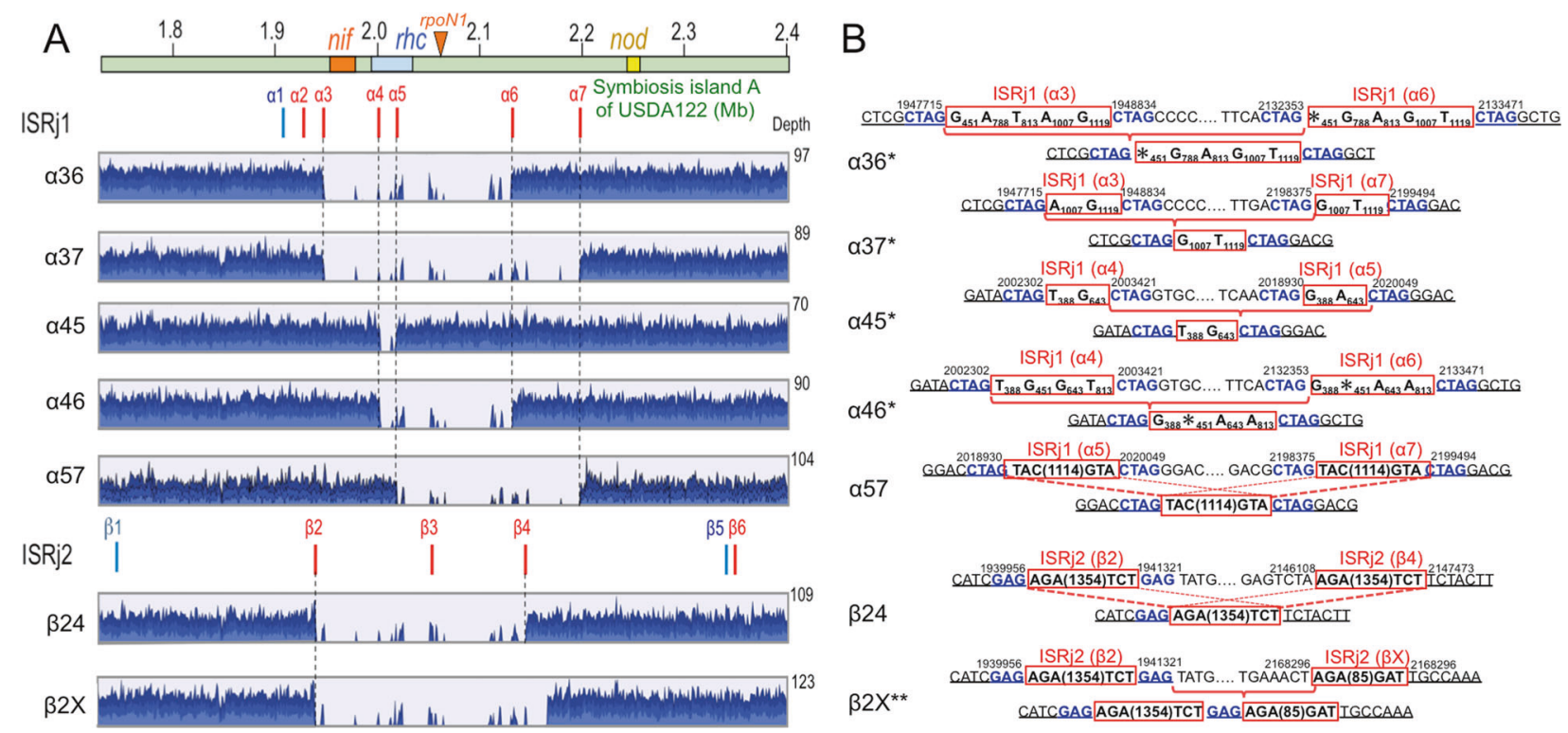

Fig. 2 Mapping profiles of MiSeq reads of $B$. diazoefficiens USDA122 mutant on the parent genome (A) and their junction sequences (B). A Representative mapping profiles of $\alpha 36, \alpha 37, \alpha 45, \alpha 46, \alpha 57, \beta 24$, and $\beta 2$ X were derived from derivatives W17, W29, W27, W46b, HG12, W35, and W20, respectively (Table S2). The colors of IS positions and gene clusters were the same in Fig. 1B. B Sequence comparisons of the junctions around the IS elements between the parent (above) and mutant (below) for respective deletion patterns of the USDA122. Red boxes (brackets) and blue letters indicate IS elements and putative target duplication sites, respectively. When DNA sequences of the two IS elements were slightly different, the different nucleotides within the IS elements are shown in the red IS boxes, where "*" shows nucleotide deletion. Red brake lines and solid lines show the estimations of the deletion modes of homologous recombination, by identical and slightly different (asterisked deletion pattern) sequences of the IS elements, respectively. Genomic positions on USDA122 are indicated above the DNA sequences.

The transposase genes a2-a7 were oriented clockwise (red in Fig. 1B), whereas a1 was oriented anticlockwise (blue in Fig. 1B). The previously described deletion patterns in USDA122 [31] were designated as a25 (mutants W3-2a, W8-1a, and W9-3a), a27 (W8$1 \mathrm{~b})$, and $\beta 23$ (W1-1a) (Fig. 1B), based on the above nomenclatures for ISRj1 and ISRj2 copies: i.e., a 25 is a deletion between $a 2$ and a5, a27 is a deletion between $\alpha 2$ and $a 7$, and $\beta 23$ is a deletion between $\beta 2$ and $\beta 3$.

On the basis of the assumption that the regions harboring rhc genes could be deleted between the IS copies in direct orientation by homologous recombination, we found 26 possible deletion patterns mediated by ISRj1, ISRj2, ISBj2, and ISBj12 (Fig. 1B). This suggests that an additional 23 patterns of IS-mediated deletions, other than the previously reported patterns, could occur in USDA122 SymA.

\section{Deletion mutants of $B$. diazoefficiens USDA122}

To test whether the predicted deletion patterns were generated, we inoculated USDA122 onto 158 plants of Rj2-soybean cultivar 'Hardee'. PCR analyses showed that 30 isolates from 72 spontaneous nodules lost nifH and/or rho genes but possessed nodC gene (Table S2). The remaining 42 isolates showed $\mathrm{nifH} / \mathrm{rhc} /$ nodC signals based on PCR analysis, which were not subjected to further genome analyses. However, we continued genome analyses of an isolate HG20 from one nodule of 'Hardee' inoculated with 122GFP (Table S3), although HG20 showed positive PCR signals of nifH/ rhcJ/nodC (Table S2). The mapping of MiSeq reads on the USDA122 genome suggested 6 new patterns of genome deletions ( $a 36, a 37, a 45, a 46, a 57$, and $\beta 24$ ) between ISRj1 or ISRj2 copies in direct orientation (clockwise), and a $\beta 2 X$ pattern between an $85-$ bp short fragment $(\beta X)$ and full copy ( $\beta 2$ ) of ISRj2 (Fig. 2A), while the remaining 12 mutants showed the previously identified deletion patterns, $\alpha 25, \alpha 27$, and $\beta 23$ (Table S2).

When the deletion junctions of the isolates were sequenced, the patterns $a 36, a 37, a 45, a 46, a 57$, and $\beta 24$ were confirmed as new deletion patterns (Fig. $2 A$ ) that were generated by typical homologous recombination between two copies of the same IS elements in direct orientation (Fig. 2B). Because four ISRj1 copies at positions a3, a4, a6, and a7 on USDA122 SymA possessed minor sequence variations and target duplicates, deletion patterns a36, a37, a45, and a46 consistently kept this sequence variation and a target duplicate (5'-CTAG) during homologous recombination (Fig. 2B). The $\beta 24$ deletion pattern also showed similar homologous recombination between two ISRj2 copies, at positions $\beta 2$ and $\beta 4$, on USDA122 SymA (Fig. 2B). All the deletion patterns, except $\beta 2 X$ (Fig. 2A), were predicted in the bioinformatic analysis, validating the prediction method (Fig. 1B).

\section{Nodulation and $\mathrm{N}_{\mathbf{2}}$ fixation of USDA122 mutants}

Among the seven newly identified deletion patterns (Fig. 2A), nif and $r h c$ clusters were deleted in $a 36, a 37, \beta 24$, and $\beta 2 X$, whereas only the rhc cluster was deleted in $a 45, a 46$, and a57 (Fig. 2A). To determine the symbiotic phenotypes of representative mutants (W42a, W29, W27, W46b, HG12, W49, and W20) covering the seven deletion patterns were inoculated onto "Hardee". All plants inoculated with the mutants were well nodulated (Fig. 3A). In contrast, wild-type USDA122 did not form nodules on Rj2-soybean roots due to Rj2-incompatibility (Fig. $3 \mathrm{~A}$ ). Then, we evaluated the $\mathrm{N}_{2}$-fixing activity of the nodules by measuring acetylene-reducing activity (ARA). No ARA was detected in the nodules infected with the mutants W42b (deletion pattern, a36), W29 (a37), W49 ( $\beta 24)$, or W20 $(\beta 2 \mathrm{X})$ (Fig. 3A), which lacked nif genes as well as $r$ c genes (Fig. 2A). ARA was detected in nodules with W27 (a45), W46b (a46), and HG12 (a57) (Fig. 3A), which had a conserved nif cluster but deleted rhc cluster (Fig. 1B); however, ARA values of W46b (a46) and HG12 (a57) were significantly lower than those of W27 (a45) (Fig. 3A). This finding indicates that genes for efficient symbiotic $N_{2}$ fixation may be located in the regions between $a 5$ and $a 6$ of USDA122 SymA (Fig. 2A). Our survey of this region suggests that $r p o N 1$ encoding sigma54 of RNA polymerase is a candidate gene for efficient symbiotic $\mathrm{N}_{2}$ fixation (Fig. 2A), because the rpoN1 mutant of $B$. diazoefficiens USDA110 shows reduced $\mathrm{N}_{2}$-fixing activity [38]. 

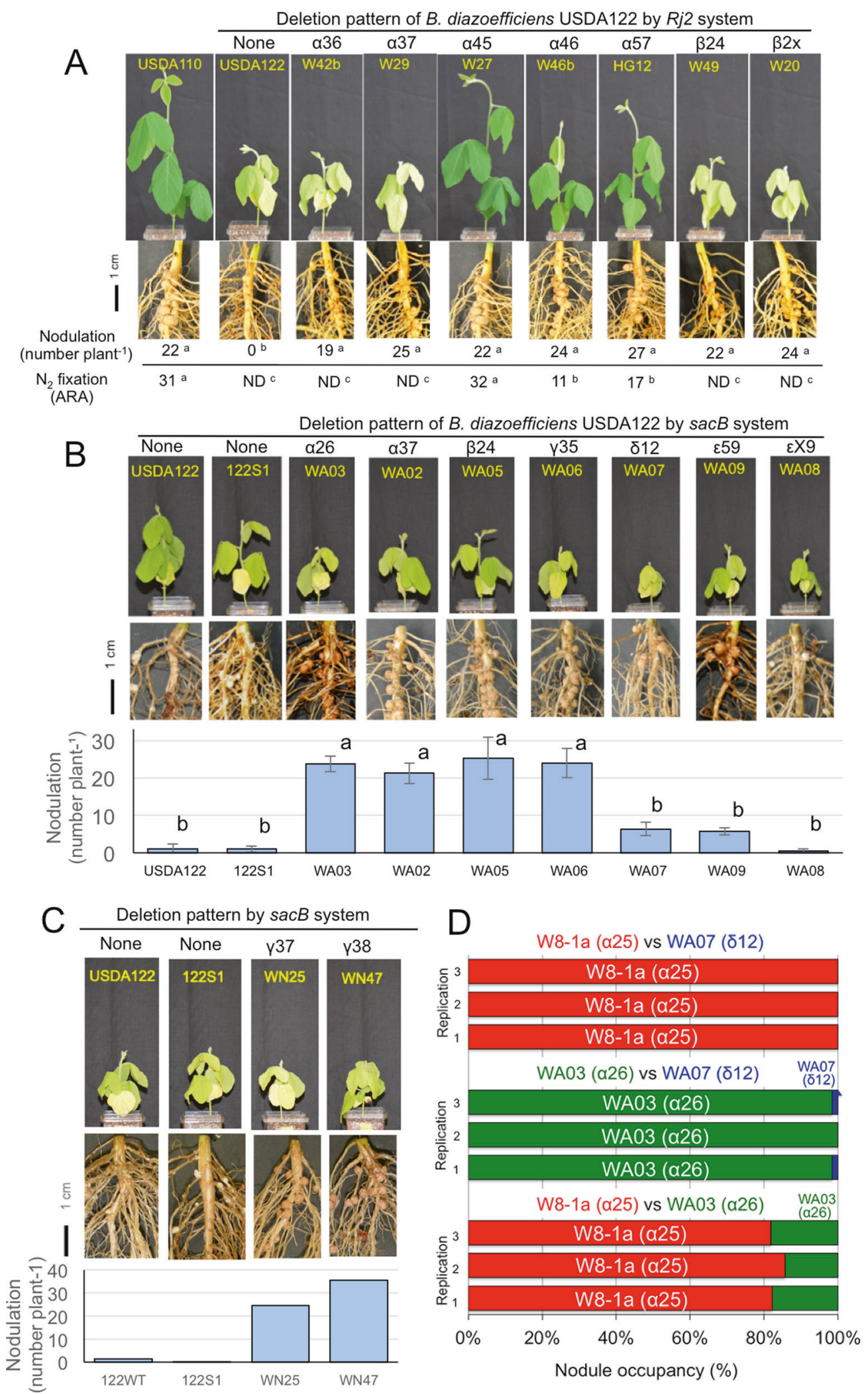

Deletion in B. diazoefficiens USDA110 and B. japonicum J5 When IS elements of $B$. diazoefficiens USDA110 [11] and $B$. japonicum J5 [39] were searched using the same strategy as that used for USDA122 above, we identified 16 and 14 different IS elements, respectively, in their genomes (Table S1); these IS elements were concentrated on SymA in both genomes (Fig. S1) Based on the location and orientation of these IS elements and the rhc cluster, we predicted 23 and 17 patterns for IS-mediated deletions on SymA of the USDA110 and J5 genomes, respectively (Fig. 4). Although $\mathrm{J} 5$ possesses a nopP that is incompatible with 
Fig. 3 Symbiotic phenotypes of Rj2-soybean plants inoculated with representative mutants, for the different deletion patterns of Bradyrhizobium diazoefficiens USDA122. Rj2-soybean plants were inoculated with deletion mutants by the Rj2 system (A) and by the sacB system (B, C). A, B "Nodulation" is expressed as the average number of nodules per plant. The bar shows standard deviation. $\mathrm{N}_{2}-$ fixing activities were evaluated by acetylene-reducing activity (ARA), which is expressed as $\mu \mathrm{mol}_{2} \mathrm{H}_{2}$ produced $\mathrm{h}^{-1} \mathrm{~g}$ nodule fresh weight ${ }^{-1}$. ND indicates "not detected" $\left(<0.08 \mu \mathrm{mol} \mathrm{h}{ }^{-1} \mathrm{~g}^{-1}\right)$. Values are expressed as averages of 3 or 4 replications, except for the nodulation data in panel $\mathrm{C}(n=2-3)$. Average values with the same letter are not significantly different by Tukey's HSD test $(P<0.05)$. D Nodule occupancy of $R j 2-$ soybean plants inoculated with mixtures of equal amounts of the two deletion mutants. The seeds of Rj2-soybean cv. Hardee was inoculated with one-to-one mixed cells of three pairwise combinations: (i) W8-1a (pattern $\alpha 25)$ and WA07 ( $\delta 12)$, (ii) WA03 ( $\alpha 26)$ and WA07 ( $\delta 12)$, and (iii) W8-1a $(\alpha 25)$ \& WA03 ( $\alpha 26)$. Nodule occupancy was determined by multiplex PCR (Fig. S8).

Rj2-soybeans, USDA110 has a nopP that is compatible with Rj2soybeans; this gene is slightly different from the USDA122 nopP [31]. Therefore, we used a USDA110 derivative that carries a USDA 122-type nopP gene (strain 110 nop $P_{122}$ ) [31] for the inoculation experiments.

After USDA110 (110nop $\left.P_{122}\right)$ and J5 were inoculated onto "Hardee", we obtained 5 and 3 mutants, respectively, from the spontaneous nodules (Table 1, Table S2). Mapping and sequencing data for these mutants indicated that the deleted regions included $r h c$ genes and were between two copies of the same IS (ISRj1 or ISBj2) in direct orientation on SymA (Black bold lines in Fig. 4). The deletion junction sequences in USDA110 and J5 suggested homologous recombination events like USDA122 (Fig. S2).

The USDA110 deletions showed four patterns (a25D, a34D, a35D, and a36D) (Fig. 4A, Table S2), all of which deleted both nif and rhc clusters (Fig. 4A). The J5 deletions showed two patterns (a34J and ү69J) (Fig. 4B, Table S2): in the a34J pattern, only the rhc cluster was deleted; but in the $169 \mathrm{~J}$ pattern, both nif and $r \mathrm{rc}$ clusters were deleted (Fig. 4B). The pattern $\mathrm{y69 \textrm {J }}$ in J5 showed the largest deletion (541 kb), which included $54 \%$ of SymA (998 kb) and $5.3 \%$ of the entire J5 genome (10.1 Mb) [39].

Symbiotic phenotypes of representative mutants from USDA110 and $\mathrm{J} 5$ were also examined by Rj2-soybean inoculation (Fig. S3). USDA110 mutants, M7 (deletion pattern, a35D), M8 (a36D), M11b (a25D), and M14 (a34D) showed a negative $\mathrm{N}_{2}$-fixing ( $\mathrm{Fix}-$ ) phenotype (Fig. S3A), as expected from their nif deletion genotype (Table 1). J5 mutants J2a ( $169 \mathrm{~J})$ and $\mathrm{J9}$ (a34J) showed Fix- and positive $\mathrm{N}_{2}$-fixing (Fix + ) phenotypes, respectively, also in accordance with their nif genotypes (Fig. S3B).

\section{IS-mediated deletion assayed using the sacB system}

We then examined whether IS-mediated deletions of the USDA122 genome can occur in free-living growth without the Rj2-soybean host. Thus, we designed an experimental system (sacB system) using a negative selection marker, $s a c B$, to detect the deletion of the rhc gene cluster (Fig. 1C). A marker cassette containing $s a c B$ and $a a d A$ genes with constitutive promoters was inserted into the intergenic region of rhc gene cluster of USDA122, resulting in strain $122 \mathrm{~S} 1$ (Fig. 1C). Because the expression of $s a c B$ encoding levansucrase is lethal for bacteria in the presence of sucrose [40, 41], we expected that sucrose-resistant colonies would have a deleted or mutagenized $\triangle a c B$ gene and that a large subset of these colonies would display a deleted rhc genes. In contrast, since aadA gene confers resistance to spectinomycin and streptomycin (hereafter, $\mathrm{Sp} / \mathrm{Sm}$ ) [42], we expected that colonies resistant to $\mathrm{Sp} / \mathrm{Sm}$ would have an intact aadA gene indicating that the rhc cluster was not deleted.

After cultivation of strain 122S1 in HM broth for 5 days, we obtained 32 sucrose-resistant mutants from independent colonies on $\mathrm{HM}$ agar plates supplemented with $10 \%(\mathrm{w} / \mathrm{v})$ sucrose. Of the 32 mutants, 22 were also sensitive to $\mathrm{Sp} / \mathrm{Sm}$ and produced no sacB PCR products (Table 2, Fig. S4), indicating that the marker cassette was likely deleted. Mapping analyses of these 22 mutants on USDA122 genome (Fig. S5A), indicated that 21 mutants were represented in ten patterns of IS-mediated deletions (a26, a36, a37, $\beta 23, \beta 24, \gamma 35, \gamma 37, \gamma 38, \delta 12$, and $\varepsilon 59)$ and one mutant, WA08, was not $(\varepsilon X 9)$ (Table S2). Sequencing verified that the deletion events involved homologous recombination via ISs in the 21 mutants, although the sequence (1081 bp) for homologous recombination of WA09 was not a full sequence of ISFK1 (2592 bp) (Fig. S5B).

Of the 32 sucrose-resistant mutants, ten were resistant to $\mathrm{Sp} / \mathrm{Sm}$ (Table 2), suggesting the existence of an intact aadA gene in sacB/ aad $A$ cassette (Fig. 1C). Subsequent analyses of $s a c B$ indicated that 8 of these mutants possessed single nucleotide polymorphisms (SNPs) on sacB (Table 2, Table S4), which may lose their levansucrase activity. Combining the two bioassays for sucrose resistance and $\mathrm{Sp} / \mathrm{Sm}$ sensitivity of $122 \mathrm{~S} 1$ greatly facilitated the efficient detection of the 21 mutants with IS-mediated deletions. In addition, the frequency of IS-mediated deletion mutants reached $2.2 \times 10^{-4}$, based on total colony-forming units in 5day-old cultures of $122 \mathrm{~S} 1$ (Table 2).

To examine the real diversity in the deletion patterns of mutants from free-living cultures, we further isolated an additional 96 sucrose-resistant mutants from 4-day-old cultures of $122 \mathrm{~S} 1$. Sp/Sm sensitivity assay suggested that 59 mutants had deleted the marker cassette. The IS-PCR analysis indicated that 49 of these 59 mutants (WN01, WN03-WN24, WN26-WN46, WN48, WN49, and WN51-WN53) had already-known deletion patterns ( $a 25, a 26, a 27, a 36, a 37, a 45, \beta 23, \beta 24, \gamma 35$, and $\delta 12$ ) (Table S2). MiSeq reads of the remaining 10 mutants were mapped on the USDA122 genome, and 4 mutants (WN02, WN25, WN47, and WN50) were identified as having new ISBj2-mediated deletion patterns ( $\gamma 37$ and $\gamma 38$ ) on USDA122 SymA (Fig. S5. Table 1, Table S2).

The remaining six mutants (WN101-WN106) showed complicated mapping profiles based on the USDA122 genome (Table S2, Fig. S6). The deletion events likely occurred between a full IS copy (ISRj2 or ISBj2) and a shorter fragment of the corresponding IS in three mutants, WN101, WN102, and WN103 (Fig. S6). MiSeq reads of mutant WN105 showed two characteristic loci of heavily (74 kb) and null $(145 \mathrm{~kb})$ mapped regions that were adjacent to each other (Fig. 5A). The heavily mapped region (mapping depth, $\sim 4$ times the basal level) had an ISFK1 fragment at each border. The results for mutant WN105 suggest that multiple duplication events likely occurred between two ISFK1 fragments, as well as a deletion event (detected as the $145 \mathrm{~kb}$ null mapped region), that included nif/rhc gene clusters (Fig. 5A).

\section{Symbiotic phenotype of deletion mutants isolated by the sacB} system

Representative mutants from $s a c B$ system were inoculated onto "Hardee" (Fig. 3B, C). The mutants WA03 (deletion pattern, a26), WA02 (a37), WA05 ( 324 ), and WA06 ( $\gamma 35$ ) produced large numbers of nodules, in contrast to the parent strain, 122S1. However, WA07 ( $\delta 12)$ and WA09 (ع59) produced significantly lower numbers of nodules than the above mutants (Fig. $3 \mathrm{~B}$ ). In a survey for genes relevant to nodulation, nodVW gene, which encodes a flavonoid sensor and regulator [43] was found in the deleted regions of WA07 ( 812$)$ and WA09 (ع59) (Fig. S5A). Thus, it is possible that these mutants were not obtained in the experiments involving inoculation of Rj2-soybean 'Hardee' plants (hereafter, Rj2-system), due to the lack of nodVW. 
A Symbiosis island $A$ of USDA110 genome $(\mathrm{Mb})$

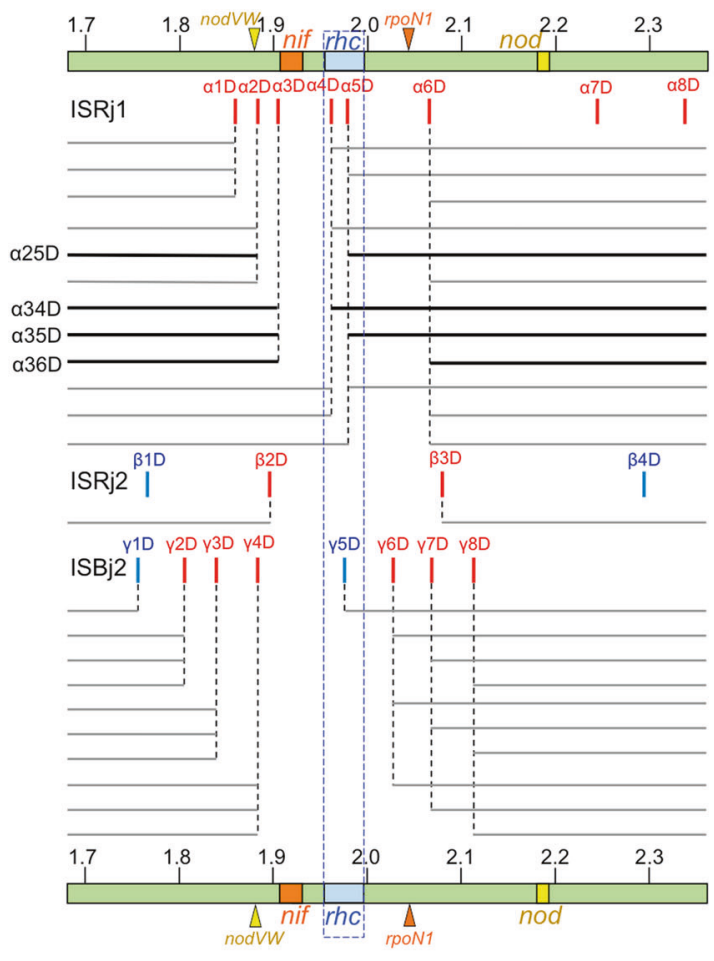

B Symbiosis island $\mathrm{A}$ of $\mathrm{J} 5$ genome $(\mathrm{Mb})$

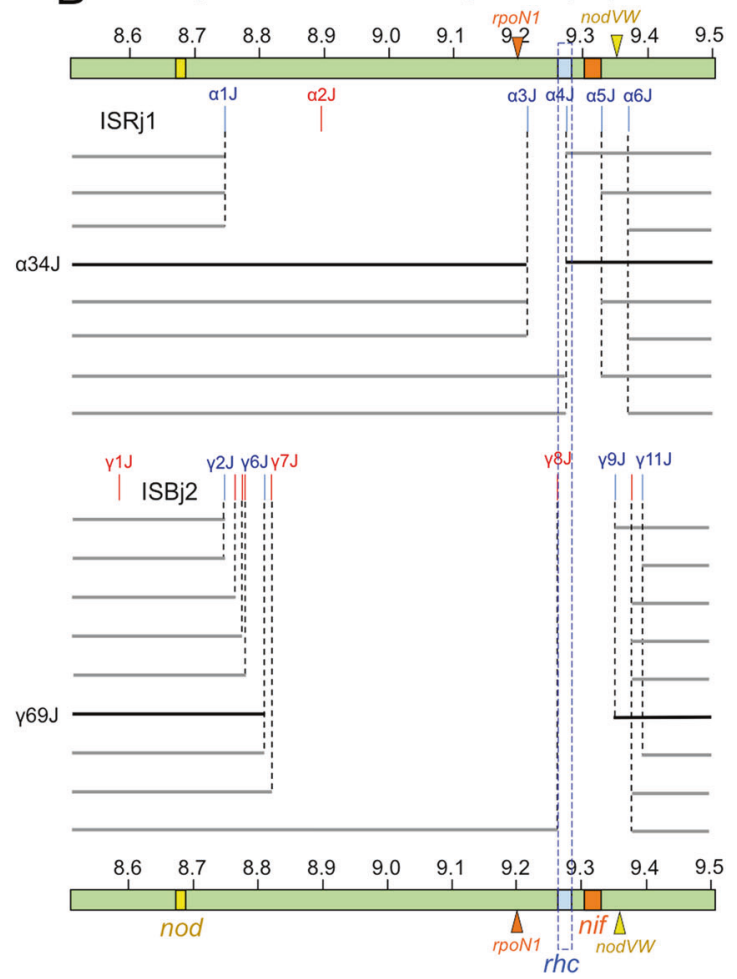

Fig. 4 The positions of insertion sequences (ISs) and the predicted IS-mediated deletion patterns within symbiosis island $A$ of Bradyrhizobium diazoefficiens USDA110 (A) and B. japonicum J5 (B). Red and blue vertical lines show ISs with clockwise and anticlockwise directions on the genomes, respectively. Predicted deletion patterns between the ISs were estimated on the same assumption as in Fig. $1 \mathrm{~A}$ and B. The deletion patterns were named based on the IS positions, such as $\alpha 25 \mathrm{D}$ for USDA 110 and $\alpha 34 \mathrm{~J}$ for J5. Other indications are identical to those in Fig. 1.

\section{Comparisons of deletion patterns obtained by the $R j 2$ and sacB systems}

When deletion patterns of USDA122 mutants obtained using Rj2 system were compared with those from $s a c B$ system, both systems were found to share common deletion patterns of a25, $a 27, a 36, a 37, a 45, \beta 23$, and $\beta 24$ (Table 1). However, sacB system generated unique deletion patterns $(\alpha 26, \gamma 35, \gamma 37, \gamma 38, \delta 12$, and ع59) that were not obtained from Rj2 system (Table 1); 11 and 25 mutants showed the deletion patterns of a26 and $\delta 12$, respectively, accounting for approximately half of the 74 ISmeditated mutants from sacB system (Table 1). Therefore, we designed an inoculation experiment to compare the competitive nodulation ability of $s a c B$ system-specific deletion mutants WA03 (a26) and WA07 ( $\delta 12)$ with that of a mutant detected by both systems, W8-1a (a25) (Fig. S7, Table 1).

When one-to-one mixed cells of mutants W8-1a (a25) and WA07 ( $\delta 12)$ were inoculated onto "Hardee", the nodule occupancy of W8-1a (a25) was extremely dominant against WA07 ( $\delta 12)$ (Fig. 3D). When one-to-one mixed cells of mutants WA03 (a26) and WA07 ( $\delta 12)$ were inoculated, the nodule occupancy of WA03 (a26) was also dominant against WA07 (ס12) (Fig. 3D). These results are expected, due to the low nodulation capability of mutant WA07 ( $\delta 12)$ even in single inoculation experiments (Fig. 3B). When one-to-one mixed cells of mutants W8-1a (a25) and WA03 (a26) were inoculated, the nodule occupancy of W8-1a (a25) was $80 \%$, indicating that nodulation by mutant WA03 (a26) was inferior to that by W8-1a (a25) (Fig. 3D). These results suggest that the mutants with $s a c B$-specific deletion patterns a26 and $\delta 12$ failed to efficiently nodulate soybean roots under competitive conditions during the selection process of Rj2 system, thus explaining the lack of mutants with deletion patterns a26 and $\delta 12$ obtained from Rj2 system (Table 1).

\section{PCR verification of IS-mediated deletion}

To obtain more direct evidence for IS-mediated deletions during the cultivation of free-living cells of USDA122 and 122S1, we developed two sets of PCR reactions to detect a25 and a26 deletion events mediated by ISRj1 (Fig. S8). When total DNAs from full growth cultures of USDA122 and 122S1 were used as template DNAs, we detected PCR products of $1.8-\mathrm{kb}$ for the a 25 deletion and $2.2-\mathrm{kb}$ for the a26 deletion, in both strains (Fig. S8). This result indicates that free-living cells of USDA122 and $122 S 1$ stochastically generated ISRj1-mediated deletions during simple cultivation.

\section{IS-mediated duplications on symbiosis islands}

Our sacB and Rj2 systems are not designed to detect duplication events on symbiosis islands; however, by chance, a mutant in which both duplication and deletion happened simultaneously, mutant WN105, was identified (Fig. 5A). Therefore, we explored ISmediated duplications on symbiosis islands by using a culture collection of $B$. diazoefficiens from soybean fields in Hokkaido, Japan. The DNAs of 62 strains from the culture collection were sequenced on the MiSeq platform and mapped to the reference genome of $B$. diazoefficiens USDA110 [11, 44]. Two field isolates, HF07 and HH12, had heavy mapping depths in a region of SymA in the USDA110 genome, when compared with closely related isolates HF08 and $\mathrm{HH} 15$ (Fig. S9). When HF07 and HH12 were further purified by single colony isolation on $\mathrm{HM}$ agar medium to produce isolates F07S3 and $\mathrm{H} 12 \mathrm{~S} 4$, respectively, the re-isolates still showed heavy mapping depth in the same region (Fig. 5B, C). Magnification of F07S3 and H12S4 profiles showed that the heavy mapping region spanned 263 and $230-\mathrm{kb}$ of SymA, respectively, and included nif and rhc genes but not the nod genes (Fig. 5B). Interestingly, in both F07S3 and H12S4, the borders of the heavily 
Table 1. Summary of the deletion patterns in mutants of B. diazoefficiens USDA122, B. diazoefficiens USDA110, and B. japonicum J5.

\begin{tabular}{|c|c|c|c|c|c|c|c|c|c|}
\hline \multirow[t]{2}{*}{ Pattern $^{a}$} & \multirow[t]{2}{*}{ Back-ground } & \multirow[t]{2}{*}{ IS } & \multicolumn{2}{|c|}{ Number of mutants } & \multirow[t]{2}{*}{$\begin{array}{l}\text { Deleted length (\% in } \\
\text { SymA) }{ }^{\mathbf{b}}\end{array}$} & \multicolumn{2}{|c|}{$\begin{array}{l}\text { Symbiotic genes } \\
\text { deletion }\end{array}$} & \multicolumn{2}{|c|}{ Plant phenotype ${ }^{c}$} \\
\hline & & & Rj2 system & sacB system & & nif/rhc & Others & Nodulation & $\mathbf{N}_{\mathbf{2}}$-fixation \\
\hline$\alpha 25^{*}$ & USDA122 & ISRj1 & 12 & 5 & $91 \mathrm{~kb}(14 \%)$ & nif, rhe & & Normal & Fix- \\
\hline$\alpha 26$ & USDA122 & ISRj1 & & 11 & 204 kb (30\%) & nif, rhe & rpoN1 & Normal & Fix- \\
\hline$\alpha 27^{*}$ & USDA122 & ISRj1 & 3 & 3 & $270 \mathrm{~kb}(40 \%)$ & nif, rhe & rpoN1 & Normal & Fix- \\
\hline$\alpha 36$ & USDA122 & ISRj1 & 9 & 4 & 185 kb (27\%) & nif, rhc & rpoN1 & Normal & Fix- \\
\hline$\alpha 45$ & USDA122 & ISRj1 & 3 & 1 & 17 kb (2\%) & $r h c$ & & Normal & Fix + \\
\hline$\alpha 46$ & USDA122 & ISRj1 & 1 & & 130 kb (19\%) & $r h c$ & rpoN1 & Normal & $\mathrm{Fix}+/-$ \\
\hline$\alpha 57$ & USDA122 & ISRj1 & 1 & & 179 kb (27\%) & $r h c$ & rpoN1 & Normal & Fix $+/-$ \\
\hline$\beta 23^{*}$ & USDA122 & ISRj2 & 2 & 4 & 113 kb (17\%) & $r h c$ & & Normal & Fix + \\
\hline$\beta 24$ & USDA122 & ISRj2 & 3 & 7 & 206 kb (31\%) & rhc & rpoN1 & Normal & Fix- \\
\hline$\varepsilon 59$ & USDA122 & Partial ISFK1 & & 1 & 130 kb (19\%) & nif, rhe & nodVW & Low & $\mathrm{Fix}-$ \\
\hline$\alpha 34 D$ & USDA110 & ISRj1 & 1 & & 57 kb (8\%) & nif, rhe & & Normal & Fix- \\
\hline$\alpha 35 D$ & USDA110 & ISRj1 & 1 & & 74 kb (11\%) & nif, rhe & & Normal & $\mathrm{Fix}-$ \\
\hline$\alpha 36 D$ & USDA110 & ISRj1 & 1 & & 161 kb (24\%) & nif, rhe & rpoN1 & Normal & $\mathrm{Fix}-$ \\
\hline$\alpha 25 D$ & USDA110 & ISRj1 & 2 & & 96 kb (14\%) & nif, rhc & & Normal & Fix- \\
\hline$\alpha 34 J$ & $\mathrm{~J} 5$ & ISRj1 & 2 & & $61 \mathrm{~kb}(6 \%)$ & $r h c$ & & Normal & Fix + \\
\hline$\gamma 69 \mathrm{~J}$ & $\mathrm{~J} 5$ & ISBj2 & 1 & & $541 \mathrm{~kb}(54 \%)$ & nif, rhc & rpoN1 & Normal & Fix- \\
\hline
\end{tabular}

${ }^{\mathrm{a}}$ Asterisks indicate previous deletion patterns [31].

bercentage of deletion length against symbiosis island A (SymA) of USDA122 (672 kb), USDA110 (681 kb), and J5 (998kb).

${ }^{C}$ Fix phenotypes were based on Fig. 3 and Fig. S3.

Table 2. Mutation types of 32 sucrose-resistant mutants in free-living cultures of $B$. diazoefficiens $122 S 1$.

\begin{tabular}{|c|c|c|c|c|c|c|}
\hline $\begin{array}{l}\text { Antibiotics test } \\
(\mathrm{Sp} / \mathrm{Sm})(\text { Fig. S4) }\end{array}$ & $\begin{array}{l}\text { SacB PCR } \\
\text { (Fig. S4) }\end{array}$ & Mutation type & Recombination & Number of mutants & Frequency $\left(10^{-4}\right)^{b}$ & Original data \\
\hline $\begin{array}{l}\text { Sensitive } \\
(22 \text { mutants })^{c}\end{array}$ & $\begin{array}{l}\text { Negative } \\
\text { (22 mutants) }\end{array}$ & Marker cassette deletion & Non-homologous & 1 (3 \%) & 0.1 & Table S4 \\
\hline $\begin{array}{l}\text { Resistant } \\
\text { (10 mutants) }\end{array}$ & $\begin{array}{l}\text { Negative } \\
\text { ( } 2 \text { mutants) }\end{array}$ & Unknown & Unknown & $2(6 \%)$ & 0.2 & \\
\hline
\end{tabular}

${ }^{a}$ Spectinomycin (Sp) and Streptomycin (Sm)-sensitive/resistant test on agar plates (Fig. S4).

${ }^{\mathrm{b}}$ Frequency calculated from the corresponding mutant number ratio and total frequency of sucrose-resistant colonies of 122S1 that appeared at a frequency of $3.3 \times 10^{-4}$, based on CFU on HM agar plates without sucrose.

${ }^{\mathrm{C}} \mathrm{Sp} / \mathrm{Sm}$ sensitive mutants from the 32 sucrose-resistant mutants: WA01-WA10, WA12-WA19, and WA21-WA24 (Table S4). Among them, 21 were generated from deletions mediated by the full length of ISs, and 1 for a Partial ISFK1 (Table 1).

${ }^{\mathrm{d}}$ Single nucleotide polymorphisms (SNP) of the sacB gene (Table S5).

mapped regions were adjacent to copies of ISBj2: $\gamma 2 D$ and $\gamma 7 D$ in F070S3 and $\gamma 4 \mathrm{D}$ and $\gamma 8 \mathrm{D}$ in H12S4 (Fig. 4A; Fig. 5B). These results suggest that the partial duplication of the symbiosis island on the F07S3 and H12S4 genomes was meditated by ISBj2 copies.

The results of hybrid assembly of MiSeq and Nanopore reads suggest that there was tandem duplication of part of SymA: 260 $\mathrm{kb}$ in F07S3 (Fig. 5D) and $357 \mathrm{~kb}$ in H12S4 (Fig. 5E). This notion was confirmed by PCR and Sanger sequence analyses targeting the junctions of tandem duplications (Fig. 5D, E; Figs. S10 and S11). The determination of the complete genome sequences showed that the genome sizes of F07S3 $(9,432,644 \mathrm{bp})$ and H12S4 $(9,535,585 \mathrm{bp})$ were larger than those of HF08 $(9,109,292 \mathrm{bp})$ and
HH15 $(9,177,979 \mathrm{bp})$, which were obtained from the Otofuke and Kamishihoro fields, respectively, and that this increase in size was due to the tandem duplications, including nif and rhc genes on SymA (Fig. 5D, E).

\section{DISCUSSION}

The horizontal transfer events of symbiosis islands were mediated by integrative and conjugative elements (ICEs) in Azorhizobium and Mesorhizobium under laboratory conditions [13, 45, 46]. However, these studies do not address how the variations in symbiosis islands were generated during their evolution between 


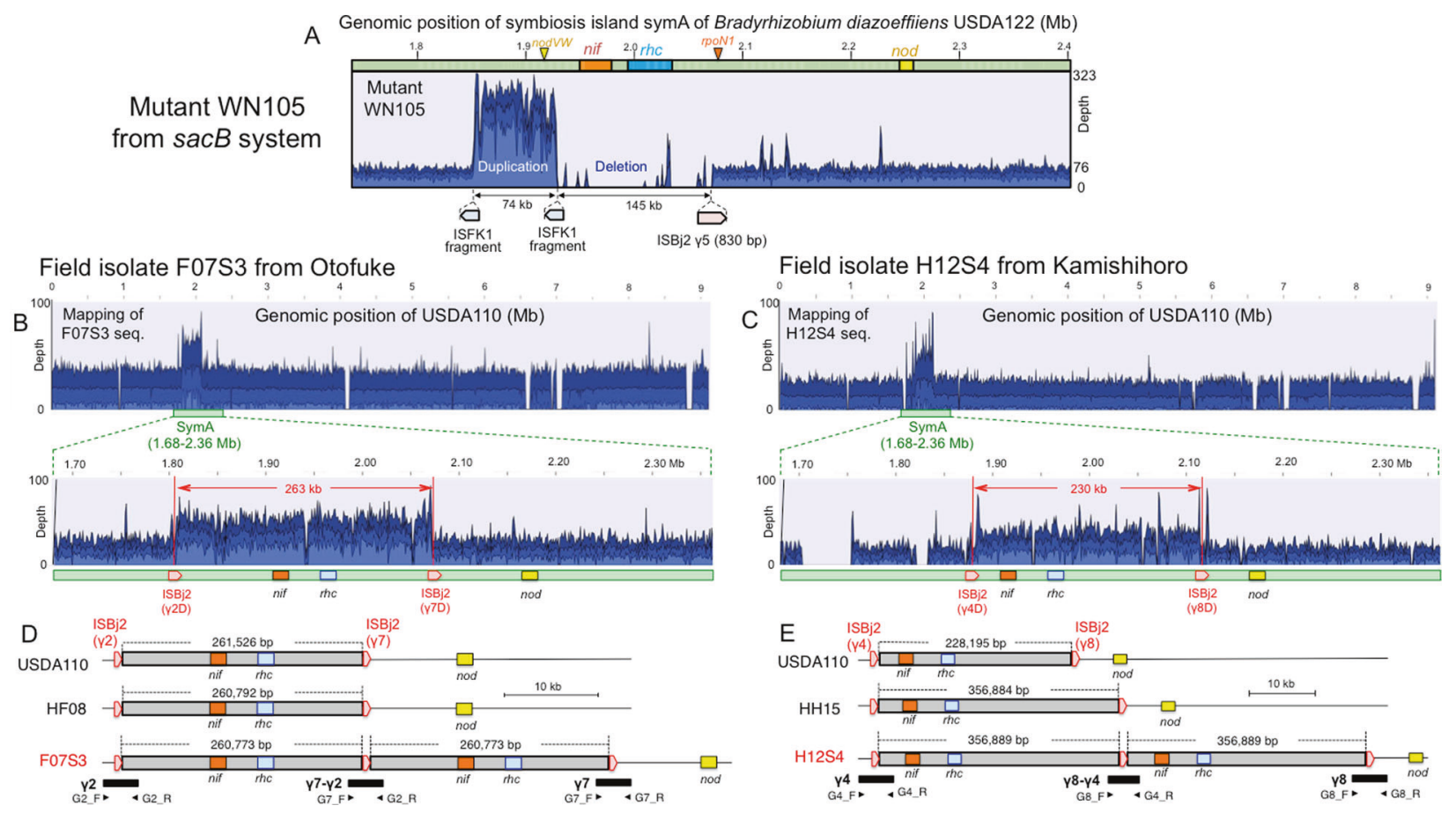

Fig. 5 IS-mediated duplication on symbiosis islands of Brdadyrhizobium diazoefficiens. A Mapping profile of the mutant WN105 from $122 \mathrm{~S} 1$ on USDA122 symbiosis island, that suggested nif/rhc deletions and duplications. (BCDE) Complete genome determination of $B$. diazoefficiens field isolates, carrying IS-mediated tandem duplications, including nif and rhc genes on the symbiosis island. B, C Mapping profiles of MiSeq reads of $B$. diazoefficiens isolates of F07S3, from Otofuke field (B) and $\mathrm{H} 12 \mathrm{~S} 4$ from Kamishihoro field (C), on the B. diazoefficines USDA110 genome. D, E Structures of tandem duplication in F07S3 (D) and H12S4 (E) mediated by different copies of ISBj2 on their symbiosis islands, which were verified by PCR sequence analyses (Figs. S10 and S11).

rhizobia and legumes [5]. Symbiosis islands of individual strains of Bradyrhizobium have become adapted to leguminous plants [47], suggesting the importance of symbiosis island evolution with host plants [5].

Recombination between IS elements in rhizobia has been known as a mechanism for generating variation in rhizobial genomes. In Sinorhizobium meliloti carrying a symbiotic plasmid, cointegrates generated IS-mediated replicon fusion, which did not demonstrate selection for the rearrangements [48]. Zhao et al. [34] reported adaptive evolution of symbiotic compatibility of Sinorhizobium fredii by inactivation of rhc and nopP genes by IS insertion. In contrast, our results demonstrate that homologous recombination between direct IS copies on bradyrhizobial symbiosis island provides a natural mechanism generating deletions, which were verified by the $R j 2$ and $s a c B$ systems on laboratory time scales. Our sequence analysis of the boundary regions of the IS-mediated deletions suggests that the major deletion events occurred via typical homologous recombination [20] between the already existing full IS copies (Fig. 2B, Fig. S2, Fig. S5B). The deletion events also occurred between the fragments of an IS element, ISFK1 (Table 1, Fig. S5B). Thus, IS-mediated deletion does not always require IS transposition by transposase activity. However, we regard the deletion pattern $\beta 2 X$ to be the trace of two successive events: ISRj2 transposition and subsequent ISRj2mediated deletion, based on border sequence analysis (Fig. 2B, Fig. S12).

Most of the IS-mediated mutants with deleted nif and rhc genes exhibited a Fix- phenotype under the genomic backgrounds of $B$. diazoefficiens USDA122 and USDA110 and $B$. japonicum J5 (Table 1). The incidence of IS-mediated deletion mutants ranged from $2.2 \times 10^{-4}$ (Tables 2) to $8.1 \times 10^{-4}$ in the experiments where 122S1 was grown free-living for 4-5 days. In addition, major deletion events following the patterns a 25 and a 26 (Table 1) were directly detected in both USDA122 and 122S1 cultures under no selection pressure by PCR (Fig. S8). Thus, these mutants defective in nif genes (non- $\mathrm{N}_{2}$ fixing mutants) were formed in subpopulations during saprophytic growth (Table 2, Fig. S8) and are likely generated even in soil environments as parasitic bradyrhizobia. However, field isolates of Bradyrhizobium from soybean nodules consistently symbiotically fixed $\mathrm{N}_{2}$ [49-51]. As for this discrepancy, one explanation may be a host sanction hypothesis to eliminate the parasitic mutants with deleted nif genes [5, 52-56]. However, little effect of host sanction was also observed by inoculation tests and modeling in the symbiosis between soybeans and Bradyrhizobium, suggesting unknown mechanisms to eliminate non- $\mathrm{N}_{2}$ fixing bradyrhizbia in field soils [57].

Previous phylogenetic analyses of a natural population of Bradyrhizobium suggested that loss-of-nodulation-capability events were potentially driven by mutations or deletions of symbiosis loci $[53,54]$. Here we found that pairs of IS copies in direct orientation were distributed around nod gene cluster on SymA of USDA122 (Fig. 1B), USDA110 (Fig. 4A), and J5 (Fig. 4B). Thus, it is possible that IS-mediated deletions of nod gene clusters may be an underlying mechanism for the loss of nodulation capabilities in natural bradyrhizobial populations $[53,54]$.

The symbiosis island structures of isolates F07S3 and H12S4 demonstrated tandem duplications of symbiosis island regions (260-357 kb) via two different copies of ISBj2 in direct orientation (Fig. 5B-E); this process appears similar to IS-mediated deletion in that the duplicated regions contained nif/rhc gene clusters between two copies of the same IS element in direct orientation. The above two isolates were found among 63 isolates of $B$. diazoefficiens in two independent fields in Hokkaido, Japan. Thus, the duplication events happened in nature, and their incidence was as high as $3 \%(2 / 63)$. Studies of the evolutionary role of IS elements in symbiotic bacteria have focused on genome reduction in obligate symbionts via IS-mediated deletion and host dependence [1-3]. On the basis of our findings, the IS- 
mediated duplication may play an important role in the evolution of rhizobia as facultative symbionts. Tandem duplications on genomes are of great importance in evolutionary genetics and cancer biology because they can dramatically alter gene functions via subsequent genome remodeling [58-60]. In addition, tandem duplications and deletions are often generated by similar recombination mechanisms [58-60]. Since we found IS-mediated tandem duplications on SymA in two field isolates (Fig. 5B-E), we consider that the structures of bradyrhizobial symbiosis islands are likely in a state of flux, and variants could be generated via tandem duplications and subsequent remodeling, including deletions and genetic exchange within rhizobial populations [58-60]. The structures of large tandem duplications in symbiotic Bradyrhizobium species have been previously overlooked due to conventional short DNA sequencing rather than long-read sequencing technologies (ideally $>400 \mathrm{~kb}$ ).

On the basis of our findings of experimental deletions and natural duplications, we propose a comprehensive hypothesis for symbiosis island evolution: (i) active ISs transpose to form IS-rich loci within low $\mathrm{G}+\mathrm{C}$ symbiosis islands; (ii) the ISs stochastically induce deletions and duplications that generate variations in the symbiosis islands, and (iii) host plants select the variants of the symbiosis islands in rhizobial populations for improved adaptations.

Extensive attention has been paid to the suppression of plant immunity to establish rhizobia-legume symbiosis [35, 61-64]. However, incompatible NopP protein in rhizobia strongly induces plant immunity via the host $R j 2$ allele, leading the host to reject the rhizobial infection $[31,64]$. A foliar systemic resistant regulator, glycerol-3-phosphate, is required for Rj2 incompatibility through root-shoot-root signaling [65]. In addition, Zhang et al. [66] reported that a new resistant protein of NNL1 gene in soybean accessions directly interacts with NopP effector from $B$. diazoefficiens USDA110 to inhibit nodulation through root hair infection. Taken together with our results, such incompatibility might facilitate co-evolution of symbiosis islands in bradyrhizobia, as a host driving force in nature.

\section{MATERIALS AND METHODS}

Bacterial materials, marker cassette construction, nodulation assay, nitrogen fixation assay, IS identification, PCR primers (Table S5), deletion profile assay, and complete genome determination are described in Supplementary materials.

\section{REFERENCES}

1. Lo WS, Huang YY, Kuo $\mathrm{CH}$. Winding paths to simplicity: genome evolution in facultative insect symbionts. FEMS Microbiol Rev. 2016;40:855-74.

2. Toft C, Andersson SG. Evolutionary microbial genomics: insights into bacterial host adaptation. Nat Rev Genet. 2010;11:465-75.

3. Batut J, Andersson SG, O'Callaghan D. The evolution of chronic infection strategies in the alpha-proteobacteria. Nat Rev Microbiol. 2004;2:933-45.

4. Poole P, Ramachandran V, Terpolilli J. Rhizobia: from saprophytes to endosymbionts. Nat Rev Microbiol. 2018;16:291-303.

5. Remigi P, Zhu J, Young JPW, Masson-Boivin C. Symbiosis within symbiosis: evolving nitrogen-fixing legume symbionts. Trends Microbiol. 2016;24:63-75.

6. Ormeño-Orrillo E, Martínez-Romero E. A genomotaxonomy view of the Bradyrhizobium genus. Front Microbiol. 2019;10:1334.

7. Garrido-Oter R, Nakano RT, Dombrowski N, Ma KW, The AgBiome Team, McHardy $A C$, et al. Modular traits of the Rhizobiales root microbiota and their evolutionary relationship with symbiotic rhizobia. Cell Host Microbe. 2018;24:155-67.

8. Avontuur JR, Palmer M, Beukes CW, Chan WY, Coetzee MPA, Blom J, et al. Genome-informed Bradyrhizobium taxonomy: where to from here? Syst Appl Microbiol. 2019;42:427-39.

9. Grönemeyer JL, Reinhold-Hurek B. Diversity of bradyrhizobia in subsahara Africa: a rich resource. Front Microbiol. 2018;9:2194.

10. Kaneko T, Maita H, Hirakawa $H$, Uchiike N, Minamisawa $K$, Watanabe $A$, et al. Complete genome sequence of the soybean symbiont Bradyrhizobium japonicum strain USDA6T. Genes (Basel). 2011;2:763-87.
11. Kaneko T, Nakamura $Y$, Sato $S$, Minamisawa K, Uchiumi T, Sasamoto $S$, et al. Complete genomic sequence of nitrogen-fixing symbiotic bacterium Bradyrhizobium japonicum USDA110. DNA Res. 2002;9:189-97.

12. Siqueira AF, Ormeño-Orrillo E, Souza RC, Rodrigues EP, Almeida LG, Barcellos FG, et al. Comparative genomics of Bradyrhizobium japonicum CPAC 15 and Bradyrhizobium diazoefficiens CPAC 7: elite model strains for understanding symbiotic performance with soybean. BMC Genomics. 2014;15:420.

13. Ling J, Wang $\mathrm{H}$, Wu $\mathrm{P}$, Li T, Tang $\mathrm{Y}$, Naseer $\mathrm{N}$, et al. Plant nodulation inducers enhance horizontal gene transfer of Azorhizobium caulinodans symbiosis island. Proc Natl Acad Sci USA. 2016;113:13875-80.

14. Uchiumi T, Ohwada T, Itakura M, Mitsui H, Nukui N, Dawadi P. et al. Expression islands clustered on the symbiosis island of the Mesorhizobium loti genome. J Bacteriol. 2004;186:2439-48.

15. Kasai-Maita H, Hirakawa H, Nakamura Y, Kaneko T, Miki K, Maruya J, et al. Commonalities and differences among symbiosis islands of three Mesorhizobium loti strains. Microbes Environ. 2013;28:275-8.

16. Sullivan JT, Trzebiatowski JR, Cruickshank RW, Gouzy J, Brown SD, Elliot RM, et al. Comparative sequence analysis of the symbiosis island of Mesorhizobium loti strain R7A. J Bacteriol. 2002;184:3086-95.

17. Perry BJ, Sullivan JT, Colombi E, Murphy RJT, Ramsay JP, Ronson CW. Symbiosis islands of Loteae-nodulating Mesorhizobium comprise three radiating lineages with concordant nod gene complements and nodulation host-range groupings. Microb. Genom. 2020;6:mgen000426.

18. Greenlon A, Chang PL, Damtew ZM, Muleta A, Carrasquilla-Garcia N, Kim D, et al. Global-level population genomics reveals differential effects of geography and phylogeny on horizontal gene transfer in soil bacteria. Proc Natl Acad Sci USA. 2019;116:15200-9.

19. Consuegra J, Gaffé J, Lenski RE, Hindré T, Barrick JE, Tenaillon O, et al. Insertionsequence-mediated mutations both promote and constrain evolvability during a long-term experiment with bacteria. Nat Commun. 2021;12:980.

20. Siguier $P$, Gourbeyre $E$, Chandler M. Bacterial insertion sequences: their genomic impact and diversity. FEMS Microbiol Rev. 2014;38:865-91.

21. Vandecraen J, Chandler M, Aertsen A, Van Houdt R. The impact of insertion sequences on bacterial genome plasticity and adaptability. Crit Rev Microbiol. 2017:43:709-30.

22. Couchoud C, Bertrand X, Valot B, Hocquet D. Deciphering the role of insertion sequences in the evolution of bacterial epidemic pathogens with panISa software. Microb. Genom. 2020;6:e000356.

23. Siguier $P$, Gourbeyre E, Varani A, Ton-Hoang B, Chandler M. Everyman's guide to bacterial insertion sequences. Microbiol. Spectr. 2015;3: MDNA3-0030-2014.

24. Caldwell BE. Inheritance of a strain-specific ineffective nodulation in soybeans. Crop Sci. 1966;6:427.

25. Hayashi M, Saeki Y, Haga M, Harada K, Kouchi H, Umehara Y. Rj (rj) genes involved in nitrogen-fixing root nodule formation in soybean. Breed Sci. 2012;61:544-53.

26. Shobudani $M$, Htwe $A Z$, Yamakawa $T$, Ishibashi $M$, Tsurumaru $H$. Mutants disrupted in the type III secretion system of Bradyrhizobium elkanii BLY3-8 overcame nodulation restriction by Rj3-genotype soybean. Microbes Environ. 2020;35:ME19151.

27. Staehelin C, Krishnan HB. Nodulation outer proteins: double-edged swords of symbiotic rhizobia. Biochem. J. 2015;470:263-74.

28. Miwa H, Okazaki S. How effectors promote beneficial interactions. Curr Opin Plant Biol. 2017;38:148-54.

29. Ratu STN, Teulet A, Miwa H, Masuda S, Nguyen HP, Yasuda M, et al. Rhizobia use a pathogenic-like effector to hijack leguminous nodulation signalling. Sci Rep. 2021;11:2034.

30. López-Baena FJ, Monreal JA, Pérez-Montaño F, Guasch-Vidal B, Bellogín RA, Vinardell JM, et al. The absence of Nops secretion in Sinorhizobium fredii $\mathrm{HH} 103$ increases GmPR1 expression in Williams soybean. Mol Plant Microbe Interact. 2009;22:1445-54.

31. Sugawara $M$, Takahashi $S$, Umehara $Y$, Iwano $H$, Tsurumaru $H$, Odake $H$, et al. Variation in bradyrhizobial NopP effector determines symbiotic incompatibility with Rj2-soybeans via effector-triggered immunity. Nat Commun. 2018;9:3139.

32. Tsukui T, Eda S, Kaneko T, Sato S, Okazaki S, Kakizaki-Chiba K, et al. The type III secretion system of Bradyrhizobium japonicum USDA122 mediates symbiotic incompatibility with Rj2 soybean. Appl Enviro Microbiol. 2013;79:1048-51.

33. Sugawara $M$, Umehara $Y$, Kaga $A$, Hayashi $M$, Ishimoto $M$, Sato $S$, et al. Symbiotic incompatibility between soybean and Bradyrhizobium arises from one amino acid determinant in soybean Rj2 protein. PLoS ONE. 2019;14: e0222469.

34. Zhao R, Liu LX, Zhang YZ, Jiao J, Cui WJ, Zhang B, et al. Adaptive evolution of rhizobial symbiotic compatibility mediated by co-evolved insertion sequences. ISME J. 2018;12:101-11.

35. Benezech C, Doudement $M$, Gourion B. Legumes tolerance to rhizobia is not always observed and not always deserved. Cell Microbiol. 2020;22:e13124. 
36. Sugawara M, Tsukui T, Kaneko T, Ohtsubo Y, Sato S, Nagata Y, et al. Complete genome sequence of Bradyrhizobium diazoefficiens USDA 122, a nitrogen-fixing soybean symbiont. Genome Announc. 2017;5:e01743-16.

37. lida T, Itakura M, Anda M, Sugawara M, Isawa T, Okubo T, et al. Symbiosis island shuffling with abundant insertion sequences in the genomes of extra-slowgrowing strains of soybean bradyrhizobia. Appl Environ Microbiol. 2015;81: 4143-54.

38. Kullik I, Fritsche S, Knobel H, Sanjuan J, Hennecke H, Fischer HM. Bradyrhizobium japonicum has two differentially regulated, functional homologs of the 054 Gene (rpoN). J Bacteriol. 1991;173:1125-38.

39. Kanehara K, Minamisawa K. Complete genome sequence of Bradyrhizobium japonicum J5, isolated from a soybean nodule in Hokkaido, Japan. Genome Announc. 2017;5:e01619-16.

40. Hynes MF, Quandt J, O'Connell MP, Pühler A. Direct selection for curing and deletion of Rhizobium plasmids using transposons carrying the Bacillus subtilis sacB gene. Gene 1989;78:111-20.

41. Gay P, Le Coq D, Steinmetz M, Ferrari E, Hoch JA. Cloning structural gene $s a c B$ which codes for exoenzyme levansucrase of Bacillus subtilis: expression of the gene in Escherichia coli. J Bacteriol. 1983;153:1424-31.

42. Hollingshead S, Vapnek D. Nucleotide sequence analysis of a gene encoding a streptomycin/spectinomycin adenylyltransferase. Plasmid. 1985;13:17-30.

43. Göttfert M, Grob P, Hennecke H. Proposed regulatory pathway encoded by the nodV and nodW genes, determinants of host specificity in Bradyrhizobium japonicum. Proc Natl Acad Sci USA. 1990;87:2680-4.

44. Hara S, Morikawa T, Wasai S, Kasahara Y, Koshiba T, Yamazaki K. et al. Identification of nitrogen-fixing Bradyrhizobium associated with roots of fieldgrown sorghum by metagenome and proteome analyses. Front Microbiol. 2019;10:407

45. Sullivan JT, Patrick HN, Lowther WL, Scott DB, Ronson CW. Nodulating strains of Rhizobium loti arise through chromosomal symbiotic gene transfer in the environment. Proc Natl Acad Sci USA. 1995;92:8985-9.

46. Haskett TL, Terpolilli JJ, Bekuma A, O'Hara GW, Sullivan JT, Wang P, et al. Assembly and transfer of tripartite integrative and conjugative genetic elements. Proc Natl Acad Sci USA. 2016;113:12268-73.

47. Parker MA. Legumes select symbiosis island sequence variants in Bradyrhizobium Mol Ecol. 2012;21:1769-78.

48. Guo X, Flores M, Mavingui P, Fuentes SI, Hernández G, Dávila G, et al. Natural genomic design in Sinorhizobium meliloti: novel genomic architectures. Genome Res. 2003;13:1810-7.

49. Itakura M, Saeki K, Omori H, Yokoyama T, Kaneko T, Tabata S, et al. Genomic comparison of Bradyrhizobium japonicum strains with different symbiotic nitrogen-fixing capabilities and other Bradyrhizobiaceae members. ISME J. 2009;3:326-39.

50. Yuan K, Reckling M, Ramirez MDA, Djedidi S, Fukuhara I, Ohyama T, et al. Characterization of rhizobia for the improvement of soybean cultivation at cold conditions in central Europe. Microbes Environ. 2020;35:1-13.

51. Habibi S, Ayubi AG, Ohkama-Ohtsu N, Sekimoto H, Yokoyama T. Genetic characterization of soybean rhizobia isolated from different ecological zones in northeastern Afghanistan. Microbes Environ. 2017;32:71-9.

52. Sachs JL, Ehinger MO, Simms EL. Origins of cheating and loss of symbiosis in wild Bradyrhizobium. J Evol Biol. 2010;23:1075-89.

53. Sachs JL, Skophammer RG, Regus JU. Evolutionary transitions in bacterial symbiosis. Proc Natl Acad Sci USA. 2011;108:10800-7.

54. Sachs JL, Russell JE, Hollowell AC. Evolutionary instability of symbiotic function in Bradyrhizobium japonicum. PLoS ONE. 2011;6:e26370.

55. Kiers ET, Rousseau RA, West SA, Denison RF. Host sanctions and the legumerhizobium mutualism. Nature 2003:425:78-81.

56. Oono R, Anderson CG, Denison RF. Failure to fix nitrogen by non-reproductive symbiotic rhizobia triggers host sanctions that reduce fitness of their reproductive clonemates. Proc Biol Sci. 2011;278:2698-703.

57. Marco DE, Carbajal JP, Cannas S, Pérez-Arnedo R, Hidalgo-Perea Á, Olivares J, et al. An experimental and modelling exploration of the host-sanction hypothesis in legume-rhizobia mutualism. J Theor Biol. 2009;259:423-33.

58. Reams $A B$, Roth JR. Mechanisms of gene duplication and amplification. Cold Spring Harb Perspect Biol. 2015;7:a016592.

59. Anderson RP, Roth JR. Tandem genetic duplications in phage and bacteria. Annu Rev Microbiol. 1977;31:473-505.

60. Bobay LM, Ochman $\mathrm{H}$. The evolution of bacterial genome architecture. Front Genet. 2017;8:72
61. Gourion B, Berrabah F, Ratet P, Stacey G. Rhizobium-legume symbioses: the crucial role of plant immunity. Trends Plant Sci. 2015;20:186-94.

62. Cao Y, Halane MK, Gassmann W, Stacey G. The role of plant innate immunity in the legume-rhizobium symbiosis. Annu Rev Plant Biol. 2017;68:535-61.

63. Nelson MS, Sadowsky MJ. Secretion systems and signal exchange between nitrogen-fixing rhizobia and legumes. Front Plant Sci. 2015;6:491.

64. Yang S, Tang F, Gao M, Krishnan HB, Zhu H. R gene-controlled host specificity in the legume-rhizobia symbiosis. Proc Natl Acad Sci USA. 2010;107:18735-40.

65. Shine MB, Gao QM, Chowda-Reddy RV, Singh AK, Kachroo P, Kachroo A. Glycerol3-phosphate mediates rhizobia-induced systemic signaling in soybean. Nat Commun. 2019;10:5303.

66. Zhang B, Wang M, Sun Y, Zhao P, Liu C, Qing K, et al. Glycine max NNL1 restricts symbiotic compatibility with widely distributed bradyrhizobia via root hair infection. Nat Plants. 2021;7:73-86.

\section{ACKNOWLEDGEMENTS}

We thank the Tokachi Federation of Agricultural Cooperative Associations for providing the $B$. diazoefficiens collection of field isolates in Hokkaido, Japan.

\section{AUTHOR CONTRIBUTIONS}

$\mathrm{HA}, \mathrm{HO}, \mathrm{MS}, \mathrm{SS}$, and KM designed the research; HA, HO, MS, SO, and KK performed the research; $\mathrm{HA}, \mathrm{HO}, \mathrm{MS}, \mathrm{RN}, \mathrm{KK}$, and $\mathrm{KM}$ analyzed the data; and $\mathrm{HA}, \mathrm{HO}, \mathrm{MS}, \mathrm{HM}$, and $\mathrm{KM}$ wrote the paper.

\section{FUNDING}

This work was supported by JSPS KAKENHI Grant no. 26252065/18H02112, NARO Bio-oriented Technology Research Advancement Institution (Research program on development of innovative technology), and JPNP18016 commissioned by the New Energy and Industrial Technology Development Organization (NEDO).

\section{COMPETING INTERESTS}

The authors declare no competing interests.

\section{ADDITIONAL INFORMATION}

Supplementary information The online version contains supplementary material available at https://doi.org/10.1038/s41396-021-01035-4.

Correspondence and requests for materials should be addressed to K.M.

Reprints and permission information is available at http://www.nature.com/ reprints

Publisher's note Springer Nature remains neutral with regard to jurisdictional claims in published maps and institutional affiliations.

Open Access This article is licensed under a Creative Commons Attribution 4.0 International License, which permits use, sharing, adaptation, distribution and reproduction in any medium or format, as long as you give appropriate credit to the original author(s) and the source, provide a link to the Creative Commons license, and indicate if changes were made. The images or other third party material in this article are included in the article's Creative Commons license, unless indicated otherwise in a credit line to the material. If material is not included in the article's Creative Commons license and your intended use is not permitted by statutory regulation or exceeds the permitted use, you will need to obtain permission directly from the copyright holder. To view a copy of this license, visit http://creativecommons. org/licenses/by/4.0/.

(C) The Author(s) 2021 